\title{
Correction: Isocitrate dehydrogenase 2 protects mice from high-fat diet-induced metabolic stress by limiting oxidative damage to the mitochondria from brown adipose tissue
}

Jae-Ho Lee, Younghoon Go, Do-Young Kim, Sun Hee Lee, Ok-Hee Kim, Yong Hyun Jeon, Taeg Kyu Kwon, Jae-Hoon Bae, Dae-Kyu Song (1), Im Joo Rhyu, In-Kyu Lee, Minho Shong, Byung-Chul Oh, Christopher Petucci, Jeen-Woo Park, Timothy F. Osborne and Seung-Soon Im (1)

Correction to: Experimental \& Molecular Medicine https://doi.org/10.1038/s12276-020-0379-z published online 3 February 2020

After the online publication of this article, the authors noticed an error in the Acknowledgments section. The correct statement of this article should read as follows.

The authors wish to change grant number HR18C0012 to HI14C1324. Please add "This study was funded by the
Ministry of Health \& Welfare, Republic of Korea (HI14C1324) to S.S.I." in the Acknowledgments.

The authors apologize for any inconvenience caused.

Published online: 15 June 2020 